\title{
Peces abisales de la "Triple Unión”, lugar de contacto de las placas Antártica, Sudamericana y de Nazca (archipiélago patagónico noroccidental)
}

\author{
Abyssal fishes of "Triple Union”, join point of Antarctic, South America and \\ Nazca plates (northwestern Patagonic archipelago) \\ Pablo R. Reyes ${ }^{1}$ \\ ${ }^{1}$ Falkland Islands Government, Fisheries Department. P.O. Box 598 F.I.P.A.S.S. Stanley, Falkland Islands, U.K. \\ preyes@fisheries.gov.fk
}

\begin{abstract}
A group of fishes obtained at 2000 meters depth at Penas Gulf $\left(47^{\circ} 31^{\prime} \mathrm{S}-75^{\circ} 15^{\prime} \mathrm{W}\right)$, on the so called “Triple Union”, joining point of the Antarctic, South America and Nazca plates (northwestern Patagonic archipelago), is analyzed. The fishes studied characterize this region as a high diversity environment, in which Antarctic fishes, fishes with Gondwanic distribution, northern invaders of austral origin and Atlantic invaders, come together. In the present study we give meristic and morphometric analyses of studied fishes; additional information about: Diastobranchus capensis Barnard, 1923, a species recently discovered in the southwestern Pacific; Muraenolepis microps Lönnberg, 1905 (its confirmation in Chilean Patagonia after 102 years without registry, explaining the scientific uncertainty about its presence outside Antarctic Convergence limit); a bathymetry record for Pseudoxenomystax albescens (Barnard, 1923), which provides new insigth to understand its presence in the South African and South American coasts; the extension of boreal geographic distribution of Cottunculus granulosus Karrer, 1968 in almost $1000 \mathrm{~km}$ within the Eastern South American cone; the extension of the austral distribution of Psychrolutes sio Nelson, 1980 towards Patagonian territory in $1600 \mathrm{~km}$ and the most southern record of the Psychrolutes genus; the finding of Ophthalmolycus chilensis Anderson, 1992, second specimen known for the species, extending its austral distribution in $3400 \mathrm{~km}$. With the information obtained we made a biogeographical analysis of the studied sector, catalogued into the Southeastern Pacific as a zone with strong deficits in its ichthyological knowledge.
\end{abstract}

Key words: Cottoidei, Synaphobranchidae, Ophtalmolycus, Muraenolepididae, Congridae
Resumen.- Se analiza un grupo de peces obtenidos a 2000 $\mathrm{m}$ de profundidad en el talud continental profundo del Golfo

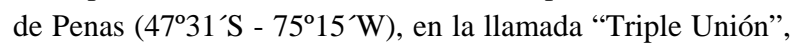
sector norte del archipiélago patagónico occidental, lugar donde se produce el contacto de las placas Antártica, Sudamericana y Nazca. El ensamble permite caracterizar al sector como un ambiente de amplia diversidad, en el que confluyen peces antárticos, de distribución gondwánica, invasores septentrionales de avanzada austral e invasores del Atlántico. En el presente estudio se entregan análisis morfológicos de los ejemplares estudiados, junto a nuevos antecedentes de Diastobranchus capensis Barnard, 1923, especie recientemente registrada en el Pacífico suroeste; la confirmación de Muraenolepis microps Lönnberg, 1905 en la Patagonia chilena tras 102 años sin registro, lo que dilucida la incertidumbre científica de su presencia fuera de los limites de la Convergencia Antártica; un registro batimétrico récord para Pseudoxenomystax albescens (Barnard, 1923), que proporciona herramientas para la comprensión de su presencia en África del Sur y Sudamérica; la ampliación de la distribución geográfica boreal de Cottunculus granulosus Karrer, 1968 en 1000 km dentro del cono sudamericano; la extensión de la distribución austral de Psychrolutes sio Nelson, 1980, en $1600 \mathrm{~km}$ hacia territorio patagónico y el registro más austral del género, que junto al registro más austral para el género Psychrolutes; el hallazgo de un ejemplar de Ophthalmolycus chilensis Anderson, 1992, el segundo conocido para la especie, ampliando su distribución austral en $3400 \mathrm{~km}$. Con los antecedentes se realizó un análisis biogeográfico del sector, catalogado dentro del Pacífico suroriental como una zona en extremo deficitaria en conocimiento ictiológico.

Palabras clave: Cottoidei, Synaphobranchidae, Ophtalmolycus, Muraenolepididae, Congridae 


\section{Introducción}

El talud continental ubicado frente a Chile (Pacífico suroriental) ha sido caracterizado por la presencia de dos regiones batimétricas con implicancias biogeográficas para la fauna íctica: un talud continental superior que se extiende aproximadamente desde los $200 \mathrm{~m}$ hasta los $1000 \mathrm{~m}$ de profundidad, y un talud continental inferior que se extiende desde los $1000 \mathrm{~m}$ a los $3000 \mathrm{~m}$, aproximadamente (Pequeño 2000).

Históricamente los esfuerzos por conocer la fauna íctica del talud continental se han centrado en su porción superior, particularmente al norte de los $40^{\circ} \mathrm{S}$ (de Buen 1959, Kong et al. 1986, Kong \& Meléndez 1991, Meléndez \& Kong 2000, Meléndez \& Meneses 1989, Oyarzún et al. 1993, Pequeño 1975, Sielfeld \& Vargas 1996, Sielfeld et al. 1995, Yañez \& Barbieri 1974).

En el sector del archipiélago patagónico occidental, en comparación con el sector boreal e insular de Chile, han existido pocos esfuerzos por mejorar el conocimiento de la ictiofauna del talud continental, y la mayoría de ellos han provenido de expediciones extranjeras de antigua data o se han centrado en el sector superior de dicho ambiente (Günther 1887, Nakamura et al. 1986, Norman 1937, Ojeda 1983, Thompson 1916). El único trabajo publicado detinado a conocer el talud continental inferior de la zona austral de Chile se enfocó en el segmento inicial de dicho ambiente, con incursiones hasta los 1353 m (Meléndez 1999), lo que confirma que el conocimiento del sector es aún precario. En este momento, de hecho, los peces del talud continental inferior al sur del paralelo $40^{\circ} \mathrm{S}$ son prácticamente desconocidos en el Pacífico suroriental y el sector se presenta como una zona en extremo deficitaria en conocimiento ictiológico, por ello se ha considerado prioritario comunicar cualquier información que aporte nuevos antecedentes sobre la ictiofauna del sector (Pequeño 2000).

A estos hechos se debe agregar que hasta la fecha la biogeografía de los peces abisales de Chile es desconocida, debido a que los estudios zoogeográficos de peces marinos chilenos y patagónicos se han enfocado básicamente a peces litorales (Lloris \& Rucabado 1991, Pequeño 1999, Sielfeld \& Vargas 1999, Ojeda et al. 2000).
Un antecedente conocido respecto a biogeografía abisal es que muchas especies de peces de aguas profundas poseen amplia distribución geográfica (Castle 1986b, Kong \& Meléndez 1991, Palmer 1986, Paxton 1999). Sin embargo, las cuencas oceánicas que albergan dichos peces abisales poseen cordilleras submarinas y zonas de subducción entre placas tectónicas, las que actúan como barreras naturales, restringiendo la mezcla de las aguas y generando barreras físico-químicas (salinidad-temperatura) que limitan el desplazamiento de los peces y, por lo tanto, su distribución geográfica en aguas profundas. De ahí la importancia de las placas tectónicas en la zoogeografía de los peces abisales (Helfman et al. 1997) y la importancia de las muestras analizadas en el presente estudio, ya que una de las particularidades relevantes del Golfo de Penas $\left(47^{\circ} 31^{\prime} \mathrm{S}\right.$ - $75^{\circ} 15^{\prime} \mathrm{W}$ ) es encontrarse próximo al lugar donde la dorsal de Chile converge oblicuamente con el continente, constituyéndose en un límite estructural y topográfico entre las placas Antártica, Sudamericana y de Nazca, motivo por el cual a este sector se le denomina Triple Unión $\left(47^{\circ} \mathrm{S}\right.$ $75^{\circ} \mathrm{W}$, aprox.) (Stern 2004).

El talud continental inferior del sector, a $2000 \mathrm{~m}$ de profundidad, no ha sido prospectado con anterioridad, básicamente por el clima adverso que caracteriza al Golfo, el cual impide operar con seguridad a los buques pesqueros. Por ello la posibilidad de disponer de material recolectado en el sector permite realizar un primer análisis sobre los peces abisales del talud continental inferior del archipiélago patagónico noroccidental, con el objetivo de mejorar el escaso conocimiento existente sobre los peces del sector.

\section{Material y métodos}

El material de estudio se obtuvo a través de una pesca de prospección realizada con espineles de fondo en el

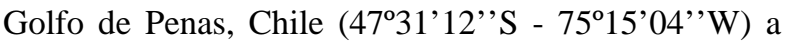
una profundidad de $2000 \mathrm{~m}$, realizada por la embarcación "Don Alfredo", dedicada a la extracción de bacalao de profundidad Dissostichus eleginoides Smitt, 1898, durante el verano del año 2003. En la oportunidad se emplearon anzuelos Mustad $\mathrm{N}^{\circ} 5$ con carnada de anchoveta Engraulis ringens Jenyns, 1842. Todas las muestras fueron fijadas en formalina al $10 \%$, rotuladas y y posteriormente remitidas al Instituto de Zoología de la Universidad Austral de Chile para su estudio y conservación. 
Las mediciones morfométricas fueron realizadas con un ictiómetro digital de $1 \mathrm{~mm}$ de precisión. La definición de los caracteres observados, las mediciones, cuantificaciones y determinación taxonómica de los ejemplares obtenidos se realizaron siguiendo los trabajos de Anderson (1992, 1994), Castle (1986a, 1986c), Cohen et al. (1990), Kong \& Meléndez (1991), Nakamura et al. (1986), Nelson $(1980,1981)$ y Tomo \& Hureau (1988).

Las abreviaturas de las mediciones corporales expresadas en porcentaje, de la longitud estándar o cefálica, utilizados en el texto corresponden a: $A B$ longitud abertura branquial; AC ancho máximo de la cabeza; AM altura mayor corporal; AMAP altura máxima aleta pectoral; AMC altura mayor de la cabeza; AMCO ancho mayor corporal; ANA altura narina anterior; ANP altura narina posterior; AO ancho oral; APC altura pedúnculo caudal; AMPC altura mínima del pedúnculo caudal; BA base de la aleta anal; BD base de la aleta dorsal; DBA distancia boca-ano; DID distancia interdorsal; DNA distancia nariz al ano; DNAH distancia narina anterior a extremo de hocico; DNPH distancia narina posterior a extremo de hocico; DO diámetro de la órbita; DON distancia ojo a narina posterior; IN ancho interorbital; LB longitud del barbo; LC longitud de la cabeza; LE longitud estándar; LFPD Longitud filamento primera aleta dorsal; LLL Longitud línea lateral; LMS longitud mandíbula superior; LMI longitud mandíbula inferior; LN longitud de la narina; LNA longitud narina anterior; LP1 longitud de la aleta pélvica; LP longitud de la aleta pectoral; LPR longitud prepectoral; LT longitud total; LOO longitud del ojo; PA longitud preanal; PD longitud predorsal; PO longitud postorbitaria; PP Longitud prepélvica; PREO longitud preoral; PRO longitud preorbitaria. Las abreviaturas de los recuentos utilizados en el texto corresponden a: A número de espinas (en números romanos) y radios (en números arábigos) de la aleta anal; BR número de branquispinas; $C$ número de espinas (en números romanos) y radios (en números arábigos) de la aleta caudal; D número de espinas (en números romanos) y radios (en números arábigos) de la aleta dorsal; ELL número de escamas sobre la línea lateral; IO número de poros interorbitales; MAND número de poros mandibulares; NAL número de poros nasales; OCC número de poros occipitales; $\mathrm{P}$ número de espinas (en números romanos) y radios (en números arábigos) de la aleta pectoral; PLL número de poros sensoriales en la línea lateral; PPO número de poros postorbitales; PRP número de poros operculares; SUB número de poros suborbitales; V número de espinas (en números romanos) y radios (en números arábigos) de la aleta pélvica. Finalmente, IZUA-PM es el acrónimo de la colección de peces marinos del Instituto de Zoología de la Universidad Austral de Chile.

Las definiciones biogeográficas de ambientes batimétricos fueron realizadas siguiendo a Pequeño (2000), las definiciones y conceptos biogeográficos latitudinales y longitudinales siguiendo a Mann (1954) y a Helfman et al. (1997). No se han considerado trabajos más recientes en el campo de la ictiogeografía de peces marinos chilenos y patagónicos (Lloris \& Rucabado 1991, Pequeño 1999, Sielfeld \& Vargas 1999, Ojeda et al. 2000) debido a que éstos se remiten básicamente a peces litorales.

\section{Resultados}

\section{Orden Anguilliformes}

Familia Congridae

\section{Pseudoxenomystax albescens (Barnard, 1923)}

\section{Material examinado}

IZUA-PM 2339, un ejemplar de 790 mm LE.

\section{Breve descripción}

Cuerpo alargado, porción anterior redondeada y la posterior comprimida, casi totalmente cubierto por cirros dérmicos, los que son más cortos en la región ventral. Cabeza pequeña. Boca pequeña, extremo posterior del maxilar alcanza aproximadamente la mitad de la órbita. Mandíbula superior ligeramente más larga que la inferior. Dientes mandibulares y de la cabeza del vómer cónicos y puntiagudos. Aleta dorsal se origina a nivel del séptimo u octavo poro de la línea lateral, ligeramente delante de la aleta pectoral.

\section{Recuentos y porcentajes de las medidas}

A 203; D 282; P 14; PLL 150. En porcentaje de la LE; LC 15.2; DO 2.5; IN 5.6; LMS 5.5; AM 8.3; BD 87.3; BA 56.9; PD 15.8; PA 43.0; LP 45.3. En porcentaje de la LC: PRO 27.2; PO 62.7; IN 20.2; DO 18.5; LP 31.1.

\section{Distribución geográfica y batimétrica}

Pacífico suroriental; Chile, desde los $32^{\circ} \mathrm{S}$ en Valparaíso hasta los $54^{\circ} \mathrm{S}$ en la Patagonia chilena (Kong \& Meléndez 1991, Nakamura et al. 1986). 
Atlántico suroccidental; Argentina y Brasil (Figueiredo et al. 2002, Nakamura et al. 1986). Atlántico suroriental; Namibia (Bianchi et al. 1993) y Sudáfrica (Castle 1986a). De 73 m a 800 m (Cervigón et al. 1980, Nakamura et al. 1986).

\section{Comentarios}

El año 1990 se describe en las inmediaciones de los montes sumergidos de Salas y Gómez a Pseudoxenomystax nielseni Karmosvkaya (1990). Ambas especies chilenas del género Pseudoxenomystax se diferencian por una serie de atributos bien descritos por Castle (1986a) y Karmosvkaya (1990), entre ellos, la cantidad de radios pectorales (16 a 18 en $P$. nielseni y 12 a 14 en $P$. albescens).

\section{Familia Synaphobranchidae}

\section{Diastobranchus capensis Barnard, 1923}

\section{Material examinado}

IZUA-PM 2338, un ejemplar de 875 mm LT.

\section{Breve descripción}

Cuerpo alargado, comprimido lateralmente. Hocico largo, puntiagudo, se extiende tras la proyección horizontal del ojo. Dos pares de narinas presentes. Las primarias adelante del ojo, frente a su sector medio. Secundarias conspicuas, tubulares, de posición subterminal en el hocico. Cuerpo desnudo, desprovisto de escamas y de textura lisa. Línea lateral sinuosa, se origina en la cabeza, cerca del sector superior del ojo, en proyección dorsal. Finaliza en el ápice de la cola, con recorrido medio por el tronco. Línea lateral provista de poros sensoriales. Mayor altura corporal presente a nivel del ano, siendo la cabeza de menor altura, al igual que la porción corporal posterior al ano, que se prolonga formando una cola larga y delgada, pero fuerte. La aleta anal es más larga que la dorsal. Ambas se originan en el tercio anterior del cuerpo y confluyen en la aleta caudal formando una sola aleta. Aleta pectoral con radios, el de mayor longitud es el más cercano al sector dorsal, los demás disminuyen progresivamente en longitud hacia el más próximo al sector ventral, que se presenta como el más corto. Pélvica ausente.

\section{Recuentos y porcentajes de las medidas}

En porcentaje de la LE: LC 16.4; LPR 14.6; LP 9.5; PA 21.6; PD 36; AM 9.3; BD 65.5; BA 58.5; AMCO 5.9.
En porcentaje de la LC: DO 13.9; IN 16.2; LMS 58.9; LMI 57.5; PRO 29.4; AO 22.9; AMC 43.9; AC 28.2; AB 20; LNA 3.9; ANA 2.7; DNAH 9.4; DNPH 21.6; ANP 3.2; DON 3.57.

\section{Distribución geográfica y batimétrica}

Circumantártico. Pacífico suroccidental; Australia y Nueva Zelanda (Paulin et al. 1989, Paxton et al. 1989). Pacífico suroriental: Chile (Reyes \& Pequeño 2006). Atlántico suroccidental: Islas Malvinas y Brasil (Brickle \& Laptikhovsky 2002, Menezes et al. 2003). Atlántico suroriental: Sudáfrica (Castle 1986c). Océano Austral: Islas Kerguelen (Gon 1990). De 850 m a 2000 m (Castle 1986c, Reyes \& Pequeño 2006).

\section{Comentarios}

El primer registro para la especie en el Pacífico Suroriental fue recientemente notificado por Reyes \& Pequeño (2006).

\section{Orden Gadiformes}

\section{Familia Muraenolepididae}

\section{Muraenolepis microps Lönnberg, 1905}

\section{Material examinado}

IZUA-PM 2336, un ejemplar de 292 mm LT.

\section{Descripción}

Cuerpo alargado y comprimido. Cabeza pequeña. Barbilla en el mentón, más larga que el diámetro del ojo. Perfil de la cabeza sobre el ojo netamente curvo. Aberturas branquiales restringidas a la zona bajo las aletas pectorales. Aletas sin espinas. Aletas dorsal, caudal y anal confluentes. Dos aletas dorsales, la primera con un solo radio, con forma de filamento, el cual no es más largo que el doble del diámetro del ojo. Aletas pélvicas en posición torácica. Escamas alargadas, no imbricadas, dispuestas en ángulo recto unas con respecto a las otras, incrustadas en la piel que parece lisa. Línea lateral arqueada sobre la aleta pectoral, se extiende posteriormente hasta poco más allá de la punta de las aletas pectorales.

\section{Recuentos y porcentajes de las medidas}

En porcentaje de la LC (57.2 mm): DO 9.4; IN 7.9. En porcentaje de la LE (279 mm): AM 17,8; LC 19,6. AB 9.6; AC 11.5; BA 51.9; BD 82.4; DID 3.9; LB 3.4; 
LFPD 8.6; LLL 26.9; LP1 26.6; LP 11.8; PA 50.2; PD 23.3; PO 94.2; PP 13.3; PRO 11.1.

\section{Distribución geográfica y batimétrica}

Océano Austral; Península Antártica y Tierra de Victoria. Islas Subantárticas; Georgia del sur, Sandwich del sur, Orcadas del sur, Shetland del sur y Maquaries. Pacífico suroriental; Chile. Atlántico suroccidental; Argentina, Islas Malvinas. De 10 m a 1600 m (Gon 1990, Fowler 1945)

\section{Comentarios}

Tomo \& Hureau (1988) y Cohen et al. (1990) consideran dudosos los registros para Sudamérica y confinan la presencia de la especie a los límites de la Convergencia Antártica. Kong \& Meléndez (1991) registran por primera vez en Chile la presencia de $M$. marmoratus Günther, 1880, especie que presenta la barbilla del mentón más corta que el diámetro del ojo, lo cual la diferencia de M. microps (Tomo \& Hureau 1988). En aguas patagónicas chilenas también se presenta M. orangiensis Vaillant, 1888 (Fowler 1945), especie que presenta el filamento dorsal tres veces más largo que el diámetro del ojo, lo cual la diferencia de M. microps (Tomo \& Hureau 1988).

\section{Orden Perciformes}

\section{Familia Zoarcidae}

\section{Ophthalmolycus chilensis Anderson 1992}

\section{Material examinado}

IZUA-PM 2337, un ejemplar de 226 mm LT.

\section{Breve descripción}

Consistencia del cuerpo firme, excepto en la parte ventral de la cabeza y el estomago. Línea lateral doble en posición medio lateral y completa, su recorrido se origina al nivel de la inserción de las aletas pectorales y concluye en el extremo posterior del cuerpo. Opérculo se extiende hasta la parte media de la base de la aleta pectoral. Labio superior continuo a través de la boca. Ojos grandes. Dientes pequeños y cónicos, presentes en vómer y palatino. Sistema de poros cefálicos bien desarrollado. Aleta dorsal confluye con la aleta caudal y la aleta anal formando una sola gran aleta continua. Aletas pectorales con radios, redondeadas. Aletas pélvicas pequeñas. Cuerpo color café homogéneo, zona ventral oscura.

\section{Recuentos y porcentajes de las medidas}

BR 6; SUB 6; PPO 4; MAND 7; NAL 2. En porcentaje de la LC (31 mm): PRO 2.74; PO 69.3; IN 1.13; DO 2.1; LP 43.5; LP1 13.5; AB 35.4; LMS 37.4; LN 6.4; AMC 48.4. En porcentaje de la LE (224 mm): LC 13.8; AM 9.8; BD 8.5; BA 6.5; PD 15.2; PA 31.7; PP 11.6; AB 4.5; AMC 6.7; AMAP 4.8.

\section{Distribución geográfica y batimétrica}

La especie es conocida por el holotipo, capturado a $1000 \mathrm{~m}$ de profundidad, frente a Tocopilla, Chile $\left(22^{\circ} 42^{\prime} \mathrm{S}-70^{\circ} 35^{\prime} \mathrm{W}\right)$ (Anderson 1992). El presente estudio amplía la distribución de O. chilensis 3400 km al sur y batimétricamente lo sitúa hasta los $2000 \mathrm{~m}$ de profundidad.

\section{Comentarios}

El género Ophthalmolycus se encuentra representado en Chile por tres especies (Pequeño 1989, 1997). Anderson (1992) diferencia a O. chilensis de los demás representantes del género por presentar aletas pélvicas, 6 branquiostegales y 4 a 5 poros postorbitales (PPO). Además, recalca el hecho que el holotipo presenta 4 PPO en su lado izquierdo y 5 PPO en su lado derecho. Esta situación no fue observada en el ejemplar IZUAPM 2337, que presentó indistintamente 4 PPO.

\section{Orden Scorpaeniformes}

\section{Familia Cuttunculidae}

\section{Cottunculus granulosus Karrer, 1968}

\section{Material examinado}

IZUA-PM 2335, un ejemplar de 185 mm LT.

\section{Breve descripción}

Espinas de la cabeza prominentes. Espinas suborbitales conspicuas, distintas y agrupadas en tres pares de parches. Ojos relativamente grandes, el diámetro ocular es igual o mayor que la distancia interorbital. Espacio interobital cóncavo. Borde dorsal de la órbita elevado. Espinas preoperculares poco desarrolladas y cóncavas. Piel sin tubérculos, relativamente firme. Primera aleta dorsal baja, espinas poco desarrolladas, bajo la piel. Segunda aleta dorsal prominente, con radios conspicuos. Aleta pectoral grande. Aletas pélvicas pequeñas. Dientes presentes en el vómer. Cuerpo de color uniformemente blanco. 


\section{Recuentos y porcentajes de las medidas}

A 9; D V-14; P 21; V 3; BR 7. En porcentaje de la LE (152 mm): LC 40.6; AC 37.2; DO 8.2; AM 23.5; IN 8.7; LMI 20.4; AMPC 4.2; LP 22.8; LP1 7.4.

\section{Distribución geográfica y batimétrica}

Plataforma continental Patagónica. Atlántico suroccidental; Islas Malvinas, Uruguay y Argentina. Pacifico suroriental; Chile. De 348 m a 800 m (Nakamura et al. 1986, Ojeda 1983)

\section{Comentarios}

Nelson \& Nakamura (1980) reconocen una alta variabilidad en los ejemplares recolectados en el Atlántico suroccidental, con la presencia de dos tipos morfológicos; una forma con espinas cefálicas relativamente largas, y una forma con espinas cefálicas cortas. El ejemplar de la muestra corresponde a la forma de espinas cefálicas relativamente largas. En Chile, la especie ha sido registrada en una ocasión, en aguas de la región de Magallanes y Antártica chilena

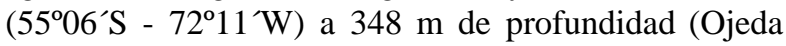
1983). El presente estudio amplía la distribución geográfica de $C$. granulosus en el Océano Pacífico suroriental $900 \mathrm{~km}$ al norte de su límite boreal conocido y confirma la presencia de la especie en Chile después de 23 años sin registros.

\section{Familia Psychrolutidae}

\section{Psychrolutes sio Nelson, 1980}

\section{Material examinado}

IZUA-PM 2340, un ejemplar de 353 mm LS.

\section{Breve descripción}

Cuerpo macizo anteriormente, se comprime y adelgaza hacia atrás desde el ano. Piel suelta, lisa y sin escamas, pero con algunos pocos cirros. Línea lateral representada por poros sensoriales más o menos separados. Cabeza grande, un poco más larga que ancha y algo redondeada. Mentón con poros tubulares. Espacio interorbital plano. Hocico y mejillas con poros tubulares que no siguen un patrón definido. Boca ancha y oblicua. Ojos más próximos al extremo anterior de la cabeza. Extremo posterior de la mandíbula alcanza la vertical trazada desde el sector medio de la órbita.

\section{Recuentos y porcentajes de las medidas}

D VII-18; A 12; V I-2; P 23. En porcentaje de la LE: DBA 47.2; AC 40; AM 31.3; APC 7.6; LP 29.2; LC 44.4; IN 16.5; LOO 5.1; DO 16.5; LMS 24.6. En porcentaje de la LC: IN 37.2; LOO 11.5; DO 37.2; LMS 55.4.

\section{Distribución geográfica y batimétrica}

Pacífico suroriental; desde $3^{\circ} \mathrm{S}$ en Perú hasta $32^{\circ} \mathrm{S}$ en Chile. De 500 a 1200 m (Nelson et al. 1985, Sielfeld \& Vargas 1996).

\section{Comentarios}

Kong \& Meléndez (1991) informan la captura de ocho ejemplares sobre el talud continental chileno, sin notificar cambios en el rango austral de distribución informado por Nelson et al. (1985). El presente estudio amplía la distribución de $P$. sio $1600 \mathrm{~km}$ al sur de su límite austral conocido.

\section{Discusión}

La presencia en Chile de Pseudoxenomystax albescens se encuentra bien documentada, e incluso se le ha caracterizado como una alternativa pesquera debido a las buenas capturas por unidad de esfuerzo (CPUE) que presenta en Chile central, a una profundidad promedio de 400 m (Arancibia 2005). Su amplia distribución geográfica y la existencia de poblaciones son aspectos que deben ser investigados (Cervigón et al. 1980). En lo concerniente a la muestra estudiada, la especie nunca ha sido notificada a una profundidad mayor de $800 \mathrm{~m}$ (Nakamura et al. 1986), por ello, el registro a $2000 \mathrm{~m}$ facilita la comprensión acerca de su amplia distribución y debe ser tomado como antecedente al momento de realizar análisis de poblaciones aisladas, tanto en Sudamérica sobre las placas de Nazca y Sudamericana, como en África del sur, sobre la placa africana.

La presencia de Diastobranchus capensis en Sudamérica ha sido detectada recientemente, en las islas Malvinas (Brickle \& Laptikhovsky 2002), Brasil (Menezes et al. 2003) y Chile (Reyes \& Pequeño 2006). Con esos aportes se extendió su distribución a todos los extremos australes de los continentes del Hemisferio Sur, con una distribución Gondwanica. Su amplia distribución en el Hemisferio puede relacionarse con aguas profundas por tratarse de una especie batidemersal (Castle 1986c) o puede vincularse a 
sistemas de montes sumergidos, como se ha observado en Nueva Zelanda, donde se la vincula con la pesquería del "orange roughy" Hoplostethus atlanticus Collet, 1889, de la cual constituye fauna acompañante con aportes importantes de biomasa (McClatchie \& Coombs 2005). Sin embargo, tal situación no ha sido reportada en la pesquería del mismo recurso efectuada en los montes sumergidos de la Zona Económica Exclusiva (ZEE) de Chile (IFOP \& UACh 2003, Oñate 2005).

La presencia de Muraenolepis microps en Tierra del Fuego, Chile e Islas Malvinas ha sido señalada por Norman (1937) quien no especifica coordenadas de captura. Fowler (1945) señala la presencia de la especie al sur de Isla Nueva y entrega coordenadas de un

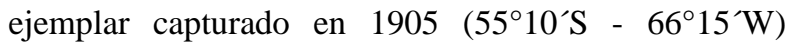
Pequeño (1989) consigna a la especie dentro de la ictiofauna chilena. Sin embargo, Tomo \& Hureau (1988) y Cohen et al. (1990) consideran los registros para Sudamérica como dudosos y confinan la presencia de la especie dentro los límites de la Convergencia Antártica. El presente estudio entrega las primeras coordenadas de captura en el Pacífico suroriental después de 102 años sin registros $\mathrm{y}$, confirma la presencia de $M$. microps en Sudamérica, fuera de los límites de la Convergencia Antártica. Además, amplía su distribución en el Océano Pacífico suroriental, 900 km aproximadamente al norte de su cuestionado límite boreal conocido. La distribución de la especie se vincula con el Océano Austral, sin embargo, algunos peces antárticos pueden movilizarse fuera de los límites de la Convergencia Antártica a través de las aguas profundas, así lo confirma la presencia en Chile y Perú de peces de origen Antártico, como el bacalao de profundidad D. eleginoides, la mollera azul Antimora rostrata (Günther, 1878) y el granadero ojisapo Macrourus holotrachys Günther, 1878, entre otros (Chirichigno 1998, Oyarzún et al. 1993, 1995, Oyarzún \& Campos 1987).

Ophthalmolycus chilensis es una especie sin registros durante los últimos 26 años, siendo éste el segundo espécimen conocido. El holotipo fue capturado frente a Tocopilla, Chile (22\%42's $70^{\circ} 35^{\prime} \mathrm{W}$ ) el año 1981. Los registros de la especie han de ser escasos debido al rango batimétrico de distribución, más allá de los 1000 m de profundidad, es decir fuera del alcance de los aparejos convencionales de pesca. Con el actual registro la especie mantiene su carácter de endémica del talud continental profundo de Chile, ubicado sobre la placa de Nazca, con un rango

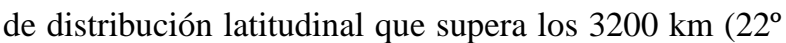
$42^{\prime} \mathrm{S}-47^{\circ} 31^{\prime} \mathrm{S}$ ) y con registros batimétricos reportados entre los $1000 \mathrm{~m}$ a $2000 \mathrm{~m}$.

La captura de un espécimen de Cottunculus granulosus en el Golfo de Penas amplía la distribución de la especie en el Pacífico suroriental 900 km aproximadamente al norte de su limite conocido

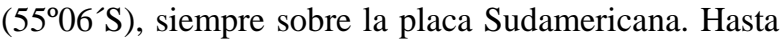
el momento la mayor cantidad de reportes de la especie se concentran en aguas del Atlántico suroccidental (Nakamura et al. 1986, Nelson \& Nakamura 1980, Wöhler et al. 1999), en cambio en el Pacífico suroriental (Chile) la especie ha sido reportada sólo en una oportunidad (Ojeda 1983). Esta situación permite asumir que el centro de distribución y abundancia de la especie se encuentra en el Atlántico sur, sobre la placa Sudamericana, y que su presencia en el Pacífico, en la zona de contacto de la placa de Nazca y Sudamericana, se encuentra limitada por factores ambientales.

Psychrolutes sio es un invasor septentrional de avanzada austral. Su presencia en el golfo de Penas amplía en 1600 km su distribución geográfica austral y lo sitúa como el representante del género con distribución más austral en el Hemisferio Sur. Pese a éste importante antecedente, hay que consignar que la especie mantiene su carácter de endémica de la placa de Nazca, donde se le conoce entre los $3^{\circ} \mathrm{S}$ y los $47^{\circ} 31^{\prime} \mathrm{S}$, es decir, a lo largo de prácticamente toda la placa.

Si a la presencia de las dos especies de Scorpaeniformes analizadas, se suman los registros en el sector, de Neophrynichthys marmoratus Regan, 1913 (Pequeño 1981) se descubre a todas las especies del complejo Cottoidei del Pacífico Suroriental viviendo en simpatría. Tal sobreposición no es conocida en ningún otro sector del planeta (Nelson 1977, Fricke 1990)

\section{Conclusiones}

Con los antecedentes expuestos se concluye que la amplia distribución geográfica de $P$. albescens y $D$. capensis podría explicarse por la presencia de larvas planctónicas leptocéfalas, capaces de ser arrastradas por las corrientes a través de grandes distancias, tal como ocurre con numerosas especies del Orden Anguilliformes (Nelson 1994). Por eso, teniendo en cuenta que la fase de leptocéfalo de $D$. capensis ha sido descrita (Castle 1969), se sugiere poner atención en los 
muestreos planctónicos en los que las larvas leptocéfalas pudieran aparecer.

O. chilensis, $P$. sio y C. granulous son especies con distribuciones localizadas dentro de Sudamérica. El actual estudio permite caracterizar dos conjuntos para estas especies: $O$. chilensis y $P$. sio que serian especies endémicas de la placa de Nazca. A su vez, $C$. granulous sería una especie endémica de la placa Sudamericana.

Respecto a $M$. microps, esta es una especie endémica del Océano Austral y placa Antártica que, sin embargo, es capaz de traspasar levemente los límites de la barrera oceanográfica que se halla en la Covergencia Antartica, presentándose en las Islas Mcquarie, ubicada en las cercanías del punto de contacto de la placa Antártica con las del Pacífico e Indo-Australiana, también ha sido registrada en las Islas Malvinas, cercanas al punto de contacto de la placa Antártica con la placa Sudamericana, finalmente, en el Golfo de Penas, lugar donde confluye la placa Antártica con las placas del Pacífico y de Nazca. Con los antecedentes se entiende que la especie es capaz de traspasar los límites de la Convergencia Antártica, sin embargo, se mantendría restringida su distribución dentro de los límites de la placa Antártica.

Hasta la fecha el escaso conocimiento indicaba para el talud continental profundo de la zona austral del Pacífico suroriental la presencia de las familias Macrouridae, Zoarcidae, Chimaeridae y Squalidae y de los órdenes Notacanthiformes y Ophidiiformes (Pequeño 2000). Con la presente investigación se agrega la presencia de los ordenes Anguilliformes, Gadiformes, Perciformes y Scorpaeniformes. Además, se confirma la presencia de la familia Zoarcidae en el sector y se agregan las familias Congridae, Synaphobranchidae, Muraenolepididae, Cottunculidae, Psychrolutidae y Nototheniidae. Material de la última familia, representada por $D$. eleginoides, fue recolectado por la embarcación en el sector pero no pudo ser estudiado debido a su condición de recurso objetivo.

Los nuevos antecedentes entregados por el presente estudio caracterizan al talud continental profundo del Golfo de Penas como un lugar de alta diversidad, en el cual peces abisales pertenecientes a diferentes grupos biogeográficos, característicos de distintas placas confluyen gracias a la presencia del punto de contacto de tres placas, Nazca, Sudamericana y Antártica.

\section{Agradecimientos}

Al Dr. Germán Pequeño del Instituto de Zoología de la Universidad Austral de Chile por sus importantes aportes bibliográficos y comentarios. Al Dr. Eric Anderson del J. L. B. Smith Institute of Ichthyology de Sudáfrica, por sus aportes bibliográficos y comentarios acerca de $O$. chilensis. A dos evaluadores anónimos por sus excelentes acotaciones. Al Sr. Tomas Neira, armador de la embarcación "Don Alfredo" por las facilidades otorgadas para el muestreo y, a los Sres. Claudio Ferrada y Francisco Pinto, en nombre de la tripulación de la embarcación mencionada, por recolectar en terreno el material de estudio.

\section{Literatura citada}

Anderson, E. 1992. Studies on the Zoarcidae (Teleostei: Perciformes) of the Southern Hemisphere. VI. Review of the genus Ophthalmolycus Regan, 1913, with description of a new species from Chile. JLB Smith Institute of Ichthyology, Special Publication 53: 1-10.

Anderson, E. 1994. Systematics and osteology of the Zoarcidae (Teleostei: Perciformes). JLB Smith Institute of Ichthyology. Ichthyology Bulletin 60,120 pp.

Arancibia, H. 2005. Alternativas pesqueras y de acuicultura para Chile central. Mundo Acuícola 24: 22-23.

Bianchi, G, K Carpenter, J .Roux, F Molloy, D Boyer \& H Boyer. 1993. FAO species identification field guide for fishery purposes. The living marine resources of Namibia. FAO, Rome. 250 pp.

Brickle P \& V Laptikhovsky. 2002. New records of deepsea fishes from the waters around the Falkland Islands. Journal of Fish Biology 60(2): 492-494.

Castle P. 1969. An index and bibliography of eel larvae. JLB Smith Institute of Ichthyology, Special Publication 7: 1121.

Castle P. 1986(a). Congridae. En: Smith MM \& PC Heemstra (eds), Smiths' sea fishes, pp.161-165. SpringerVerlag, Berlin.

Castle P. 1986b. Derichthyidae. En: Smith MM \& PC Heemstra (eds), Smiths' sea fishes, pp. 191-192. Springer-Verlag, Berlin.

Castle P. 1986(c). Synaphobranchidae. En: Smith MM \& PC Heemstra (eds), Smiths' sea fishes, pp. 188-190. SpringerVerlag, Berlin. 
Cervigón F, G Pequeño \& I Kong. 1980. Presencia de Pseudoxenomystax albescens (Barnard, 1823) y Xenomystax atrarius Gilbert, 1891, en las costas de Chile (Teleostomi, Congridae). Boletín del Museo Nacional de Historia Natural de Chile 37: 317-321.

Chirichigno N. 1998. Clave para la identificación de los peces marinos del Perú. Instituto del Mar del Perú. Segunda Edición. Publicación Especial. Callao-Perú. 496 pp.

Cohen D, T Inada, T Iwamoto \& N Scialabba. 1990. FAO species catalogue. Vol. 10. Gadiform fishes of the world (Order Gadiformes). An annotated and illustrated catalogue of cods, hakes, grenadiers and other gadiform fishes known to date. FAO Fisheries Synopsis 10 (125), 442 pp.

de Buen F. 1959. Notas preliminares sobre la fauna marina preabismal de Chile, con descripción de una familia de rayas, dos géneros y siete especies nuevos. Boletín del Museo Nacional de Historia Natural, Santiago 27(3): 173201.

Figueiredo J, A dos Santos, N Yamaguti, R Bernardes \& C Del Bianco. 2002. Peixes da Zona Econômica Exclusiva da Região Sudeste-Sul do Brasil: Levantamento com Rede de Meia-Água. 242 pp. Editora da Universidade de São Paulo, São-Paulo.

Fowler H. 1945. Fishes of Chile. Systematic Catalog (reprint). Revista Chilena de Historia Natural (I-II): 36171.

Fricke R. 1990. A new species of psychrolutid fish from Western Australia. Japan Journal of Ichthyology 36(4): 404-409.

Gon O. 1990. Synaphobranchidae. En: Gon O \& PC Heemstra (eds.), Fishes of the Southern Ocean, pp. 102104. JLB Smith Institute of Ichthyology, Grahamstown, South Africa.

Günther A. 1887. Report on the deep-sea fishes collected during the voyage of H.M.S. Challenger in the years 1873-1876. En: Report on the scientific results of the voyage of H.M.S. Challenger during the years 1873-1876. Zoology 22(57): 1-268.

Helfman G, B Collette \& D Facey. 1997. The Diversity of Fishes, 528 pp. Blackwell Science, Inc., Massachusetts

Instituto de Fomento Pesquero \& Universidad Austral de Chile. 2003. Catálogo fauna íctica asociada a la pesquería chilena de orange roughy (Hoplostethus atlanticus). Subsecretaria de Pesca. Valparaíso. 52 pp.
Karmosvkayap, E. 1990. New species of conger eels from southeastern Pacific seamounts. Journal of Ichthyology 30: 1-10.

Kong I, R Meléndez \& G Henríquez. 1986. Los peces Ophidiiformes de aguas profundas entre Arica (18 $\left.19^{\circ} \mathrm{S}\right)$ e Isla Mocha (38³0’S). Estudios Oceanológicos 7: 1-15.

Kong I \& $\mathbf{R}$ Meléndez. 1991. Estudio taxonómico y sistemático de la ictiofauna de aguas profundas capturada entre Arica e Isla Mocha (18³0' ${ }^{\circ} 38^{\circ} 30^{\prime}$ Lat. S.). Estudios Oceanológicos 10: 1-81.

Lloris D \& J Rucabado. 1991. Ictiofauna del Canal Beagle (Tierra de Fuego), aspectos ecológicos y análisis biogeográfico. Publicaciones especiales Instituto Español de Oceanografía 8, Ministerio de Agricultura, Pesca y Alimentación, Madrid. 182 pp.

Mann G. 1954. Vida de los peces en aguas chilenas. Ministerio de Agricultura e Instituto de Investigaciones Veterinarias, Universidad de Chile. Facultad de Filosofía y Educación. Santiago, Chile. 342 pp.

McClatchie S \& R Coombs. 2005. Low target strength fish in mixed species assemblages: the case of orange roughy. Fisheries Research 72:185-192.

Meléndez R. 1999. Nuevas adiciones a la ictiofauna mesopelágica y demersal entre isla Mocha y la península de Taitao (3804' $46^{\circ} 13^{\prime}$ S), Chile. Revista de Biología Marina y Oceanografía 34(2): 139-143.

Meléndez R \& I Kong. 2000. Sistemática y filogenética en peces de ambientes profundos presentes en Chile. Estudios Oceanológicos 19: 129-136.

Meléndez R \& D Meneses. 1989. Tiburones del talud continental recolectados entre Arica $\left(18^{\circ} 19^{\prime} \mathrm{S}\right)$ e Isla Mocha (38³0'S), Chile. Investigaciones Marinas, Valparaíso 17: 3-73.

Menezes N, A Buckup, L de Figueiredo \& L de Moura. (Eds) 2003. Catálogo das espécies de peixes marinhos do Brasil. Museu de Zoologia de Universidade de São Paulo. Brasil: 1-160.

Nakamura I, T Inada, M Takeda \& H Hatanaka. 1986. Important fishes trawled off Patagonia. Japan Marine Fishery Resource Research Center, Tokyo. 369 pp.

Nelson J. 1977. Fishes of the Southern Hemisfere genus Neophrynichthys (Scorpaeniformes: Cottoidei) with descriptions of two new species from New Zealand and Macquarie Island. Journal of the Royal Society of New Zealand 7(4): 485-511. 
Nelson J. 1980. Psichrolutes sio, a new psychrolutid fish (Scorpaeniformes) from the southeastern Pacific. Canadian Journal of Zoology 58(3): 443-449.

Nelson J. 1981. Two new south pacific fishes of the genus Ebinania and contributions to the systematics of Psychrolutidae (Scorpeaniformes). Canadian Journal of Zoology 60: 1470-1504.

Nelson J. 1994. Fishes of the World. 600 pp. $3^{\text {rd }}$ ed. John Wiley \& Sons, Inc. New York.

Nelson J, N Chirichigno \& F Balbontín. 1985. New material of Psichrolutes sio (Scorpaeniformes, Psychrolutidae) from the eastern Pacific of South America and comments on the taxonomy of Psichrolutes inermis and Psichrolutes macrocephalus from the eastern Atlantic of Africa. Canadian Journal of Zoology 63(2): 444-451.

Nelson J \& I Nakamura. 1980. Distribution, morphology, and taxonomy of the psychrolutid fish Cottunculus granulosus (Karrer) from the southwestern Atlantic. Canadian Journal of Zoology 58: 982-990.

Norman J. 1937. Coast fishes. part II. The Patagonian Region. Discovery Report 16: 1-150.

Ojeda P. 1983. Distribución latitudinal y batimétrica de la ictiofauna demersal del extremo austral de Chile. Revista Chilena de Historia Natural 56: 61-70.

Ojeda P, F Labra \& A Muñoz. 2000. Biogeographic patterns of Chilean littoral fishes. Revista Chilena de Historia Natural 73: 625-641.

Oñate J. 2005. Ictiofauna del sector austral de la cordillera submarina de Nazca, frente al norte de Chile y sus relaciones biogeográficas. Tesis de Biología Marina. Universidad Austral de Chile, Valdivia. 77 pp.

Oyarzún C, R Galleguillos \& J Monsalves. 1993. Variabilidad genética en el granadero de profundidad Macrourus holotrachys Günther, 1878, capturado en la zona pesquera de Talcahuano (Pisces, Gadiformes, Macrouridae). Revista de Biología Marina, Valparaíso 28(2): 331-340.

Oyarzún, C, J Monsalves \& R Galleguillos. 1995. Variabilidad en un pez de profundidad Antimora rostrata (Günther, 1878) capturado en la zona pesquera de Talcahuano (Pisces, Gadiformes, Moridae). Estudios Oceanológicos 14: 1-4.

Oyarzún C \& P Campos. 1987. Dissostichus eleginoides Smitt, 1898; consideraciones sobre su determinación taxonómica e implicancias biogeográficas (Pisces,
Perciformes, Nototheniidae). Revista de Biología Marina, Valparaíso 23(2): 173-192.

Palmer G. 1986. Trachipteridae. En: Whitehead PJP, M -L Bauchot, J -C Hureau, J Nielsen \& E Tortonese (eds.) Fishes of the north-eastern Atlantic and the Mediterranean, Vol 2: 729-732, UNESCO, Paris.

Paulin C, A Stewart, C Roberts \& P McMillan. 1989. New Zealand fish: a complete guide. National Museum of New Zealand, Miscellaneous Series 19, 279 pp.

Paxton J. 1999. Berycidae. Alfonsinos En: Carpenter KE \& $\mathrm{VH}$ Niem (eds). FAO species identification guide for fishery purposes. The living marine resources of the WCP. Vol 4. Bony fishes part 2 (Mugilidae to Carangidae): 2218-2220. FAO, Rome.

Paxton J, D Hoese, G Allen \& J Hanley. 1989. Pisces. Petromyzontidae to Carangidae. Zoological Catalogue of Australia, Vol. 7, 665 pp. Australian Government Publishing Service, Canberra.

Pequeño G. 1975. Peces del crucero "Merluza V” efectuado con el B/C “Carlos Darwin” entre Corral y Coquimbo. Enero - febrero de 1970. Boletín del Museo Nacional de Historia Natural de Chile 34: 227-232.

Pequeño G. 1981. The rare fish Neophrynichthys marmoratus Reagan 1913, in the southern slope of Chile (Teleostomi, Psychrolutidae). Journal of Natural History 15: 965-970.

Pequeño, G. 1989. Peces de Chile, Lista Revisada y Comentada. Revista de Biología Marina, Valparaíso 24(2): 1-132.

Pequeño G. 1997. Peces de Chile, Lista Revisada y Comentada: Addendum. Revista de Biología Marina y Oceanografía 32(2): 77-94.

Pequeño G. 1999. Peces del crucero CIMAR-FIORDO 2, a los canales patagónicos de Chile, con consideraciones ictiogeográficas. Ciencia y Tecnología del Mar (Chile) 22: 165-179.

Pequeño G. 2000. Delimitaciones y relaciones biogeográficas de los peces del Pacifico suroriental. Estudios Oceanológicos 19: 53-76.

Reyes P \& G Pequeño. 2006. Primer registro en el Pacífico suroriental de la anguila abisal Diastobranchus capensis arnard, 1923 (Osteicthyes: Synaphobranchidae). Revista de Biología Marina y Oceanografía 41(2): 249-253.

Sielfeld W, M Vargas \& R Fuenzalida. 1995. Peces mesopelágicos frente a la costa norte de Chile $\left(18^{\circ} 25^{\prime}\right.$ 214ㄱ'S). Investigaciones Marinas, Valparaíso 23: 83-97. 
Sielfeld W \& M Vargas. 1996. Composición y estructura de la ictiofauna demersal en la zona norte de Chile. Investigaciones Marinas, Valparaíso 24: 3-17.

Sielfeld W \& M Vargas. 1999. Review of marine fish zoogeography of Chilean Patagonia $\left(42^{\circ}-57^{\circ} S\right)$. Scientia Marina 63(1): 451-463.

Stern C. 2004. Active Andean volcanism: its geologic and tectonic setting. Revista Geológica de Chile 31(2): 161206.

Tomo A \& J Hureau. 1988. Muraenolepididae. En: Fischer W \& J Hureau (eds), Fichas FAO para la identificación de especies para los fines de pesca. Océano austral (Área de pesca 48, 58 y 88, área de la convención CCAMLR). Comisión para la Conservación de Recursos Vivos Marinos Antárticos - FAO, Roma 2: 306-319.
Thompson W. 1916. Fishes collected by the US Bureau of Fisheries Steamer “Albatross" during 1888, between Montevideo, Uruguay and Tomé, Chile, on the voyage through the Straits of Magellan. Proceedings of the United States Museum 50: 243-476.

Wöhler O, A Giussi, S García, F Sánchez, J Hansen, H.Cordo, G Alvarez, S Incorvaia, R Reta \& V Abachian. 1999. Resultados de la campaña de evaluación de peces demersales australes efectuada en el verano de 1997. INIDEP, Mar del Plata, Informe Técnico 24, 70 pp.

Yañez E \& M Barbieri. 1974. Distribución y abundancia relativa de recursos disponibles a un arte de arrastre camaronero frente a la costa de Valparaíso. Investigaciones Marinas, Valparaíso 5 (6): 137-156.

Recibido el 6 de julio de 2006 y aceptado el 16 de enero de 2007 